\title{
THE THEOREMS OF OSCILLATION OF STURM AND KLEIN. (SECOND PAPER.)
}

BY PROFESSOR MAXIME BôCHER.

(Read before the American Mathematical Society at the Meeting of February $26,1898$. )

The following pages form a continuation of $\$ \S 1,2$ of a paper presented under the same title to the Society at its December meeting and printed on pp. 295-313 of the present volume of the Bulletin. This paper will be referred to for brevity as Th. of Osc. 1. No use is here made of the results contained in $\$ 3$ of the paper just mentioned, these results being merely very special cases of some of those now given.

In $\S 1$ of the present paper, after proving two simple. theorems of Sturm, by a method different from that used by him, I have used them to throw Sturm's theorem of oscillation into a slightly generalized form. In $\$ 2$ I have proved Klein's theorem of oscillation in a very general form, although as I have restricted myself here, as in the previous paper, to the case in which the coefficients of the binomial differential equations are continuous the cases here considered are not yet the most general ones. I expect in a subsequent paper to come back to these more general cases which do not seem to present any serious difficulty.

The truth of the theorems proved in $\$ 2$ (at least in important special cases) has been suspected by Klein partly from analogy with the special cases in which the differential equation has a polynomial solution, and partly from analogy with the simple cases of Lamé's equations ( $I$ use the term here in Klein's most general sense) with two or at most three variable parameters, in which cases he had given rough geometrical proofs which however made no pretense at rigor.* In the more complicated cases no proof of any sort has as far as I know been given.

\section{\$1. Sturm's Generalized Theorem of Oscillation.}

We will assume that in the interval $a \leqq x \leqq b \quad p(x)$ and $q(x)$ are single valued continuous real functions of the real

* Cf. Klein's lithographed lectures: "Ueber lineare Differentialgleichungen der zweiten Ordnung," p. 401-431. We shall see that the restriction which Klein here makes to equations which are everywhere regular is unessential. 
variable $x$. We know (Th. of Osc. 1, (E)) that in this interval no solution of the equation :

$$
\frac{d^{2} y}{d x^{2}}+p(x) \frac{d y}{d x}+q(x) y=0
$$

which is not identically zero can have more than a finite number of roots ; and also* that, if $y_{1}$ and $y_{2}$ are two linearly independent solutions, between two successive roots of $y_{1}$ lies one and only one root of $y_{2}$.

We proceed now to a theorem which enables us to compare different solutions of the same differential equation in the same way as the first theorem of comparison ( $T h$. of Osc. 1, Theorem I) enabled us to compare corresponding solutions of different equations :

I. Assuming that $y_{1}$ and $y_{2}$ are two solutions of (1) such that $y_{2}(a) \neq 0$ and (except when $y_{1}(a)=0$ when no further assumption is necessary) $y_{1}^{\prime}(a) / y_{1}(a)>y_{2}^{\prime}(a) / y_{2}(a)$; then, if $y_{1}$ has $n$ roots $x_{i}$ such that $a<x_{1}<x_{2} \cdots<x_{n} \leqq b, y_{2}$ has just $n$ roots in the interval $a<x<x_{n}$.

For in the first place, since $y_{2}$ is clearly linearly independent of $y_{1}$, in each of the intervals $x_{i} \leqq x \leqq x_{i+1}(i=1,2, \cdots, n-1)$ $y_{2}$ has one and only one root. It is then merely necessary to prove that the same is true for the interval $a<x \leqq x_{1}$. If $y_{1}(a)=0$ this follows as before since $a$ and $x_{1}$ are then two successive roots of $y_{1}$. If $y_{1}(\alpha) \neq 0$ it is also clear at once that $y_{2}$ cannot have more than one root in the interval $a<x \stackrel{2}{\leqq} x_{1}$, as otherwise $y_{1}$ would have a root there. If then we can prove that the assumption that $y_{2}$ has no root in the interval just mentioned leads to a contradiction, our theorem will be proved. Consider the function :

$$
\bar{y}(x)=\frac{y_{1}(x)}{y_{1}(a)}-\frac{y_{2}(x)}{y_{2}(a)}
$$

which is clearly a solution of (1) linearly independent of $y_{1}$. Since $\bar{y}(a)=0$ and $\overline{y^{\prime}}(a)>0, \bar{y}(\alpha+\varepsilon)$ will, for small positive values of $\varepsilon$, be positive. Now if $y_{2}(x)$ had no root in the interval $a<x \leqq x_{1} \quad y_{2}\left(x_{1}\right)$ and $y_{2}(a)$ would have the same signs, so that $\overline{\bar{y}}\left(x_{1}\right)=-y_{2}\left(x_{1}\right) / y_{2}(\alpha)<0$, and therefore $\bar{y}(x)$ would have at least one root in the interval $a<x<x_{1}$. Therefore, since $\bar{y}(a)=0, y_{1}(x)$ would have at least one root in this interval, and this is contrary to hypothesis.

*I have recently had occasion to discuss this familiar theorem of Sturm. See BuLletin for March, 1897, p. 210. 
We come now to a proposition analogous to the second theorem of comparison (Th. of Osc. 1, Theorem II) :

II. Assuming that $y_{1}$ and $y_{2}$ are two solutions of (1) satisfying the same conditions as in Theorem $I$, and having in the interval $a<x<b$ the same number $n$ of roots ( $n$ may be zero); then if $y_{2}(b) \neq 0$ we shall have $y_{1}(b) \neq 0$ and

$$
\frac{y_{1}^{\prime}(b)}{y_{1}(b)}>\frac{y_{2}^{\prime}(b)}{y_{2}(b)} \text {. }
$$

That $y_{1}(b) \neq 0$ follows at once from Theorem I, as otherwise $y_{2}$ would have $n+1$ roots in the interval $a<x<b$. In order to prove the remainder of the theorem we will use Abel's formula :

$$
y_{2} y_{1}^{\prime}-y_{1} y_{2}^{\prime}=k e^{-\int p d x}
$$

in which $k$ is a real constant different from zero. Dividing this equation through by $y_{1} y_{2}$ we see that, except when $y_{1}$ or $y_{2}$ is zero, $y_{1}^{\prime} / y_{1}-y_{2}^{\prime} / y_{2}$ has the same sign as $k / y_{1} y_{2}$. Moreover this sign is by hypothesis positive when $x=a$, except when $y_{1}(a)=0$, in which case it is clear that for a value of $x$ a little greater than $a$ the sign is positive since it is the same as the sign of $y_{1}^{\prime} / y_{1}$. Now when $x=b$ the sign of $y_{1} y_{2}$ is the same as when $x$ is equal to or a little greater than $a$, since $y_{1}$ and $y_{2}$ change sign the same number of times in the interval $a<x<b$. Accordingly $y_{1}^{\prime} / y_{1}-y_{2}^{\prime} / y_{2}$ is positive when $x=b$, as was to be proved.

We proceed now to a generalized form of Sturm's theorem of oscillation :

III. If when $a \leqq x \leqq b$ and $L>\lambda>l^{*}, \varphi(x, \lambda)$ is a single valued continuous function of $(x, \lambda)$ and if, when $a \leqq x \leqq b$ and $L>\lambda^{\prime}>\lambda^{\prime \prime}>l, \varphi\left(x, \lambda^{\prime}\right) \geqq \varphi\left(x, \lambda^{\prime \prime}\right) \dagger ;$ if moreover

$$
\lim _{\lambda=L} \varphi(x, \lambda)=+\infty \text { and } \lim _{\lambda=l} \varphi(x, \lambda)=-\infty
$$

EXCEPT PERHAPS FOR A FINITE NUMBER OF VALUES OF $x$ IN THE INTERVAL $a \leqq x \leqq b$; then there will be one and only one value of $\lambda(L>\lambda>l)$ for which the equation:

$$
\frac{d^{2} y}{d x^{2}}=\varphi(x, \lambda) y
$$

* We may have here $L=+\infty$, or $l=-\infty$, or both $L$ and $l$ may be infinite.

†The equality sign must not hold for all values of $x$ in the interval $a \leqq x \leqq b$ (cf. Th. of Osc. 1, p. 304 and 305, footnotes). 
has a solution which has just $m$ roots in the interval $a<x<b$ and which satisfies the conditions:

$$
\begin{aligned}
& \alpha^{\prime} y(a)-a y^{\prime}(a)=0, \\
& \beta^{\prime} y(b)-\beta y^{\prime}(b)=0 .
\end{aligned}
$$

It should be noted that except for the clause printed in small capitals the theorem just stated is identical in substance (although stated in a somewhat different form) with the simple form of Sturm's theorem of oscillation (Th. of Osc. 1, Theorem VIII).* In order to prove the theorem in all its generality it is clearly sufficient to prove that :

(a) we can find a quantity $l_{1}$ such that when $l<\lambda \leqq l_{1}$ every solution of (2) has more than $m$ roots in the interval $a<x<b$; ( $\beta$ )we can find a quantity $L_{1}$ such that when $L_{1} \leqq \lambda<L$ the solution of the differential equation for which $y(\alpha)=\alpha$, $y^{\prime}(\alpha)=\alpha^{\prime}$ does not have any root in the interval $\alpha<x \leqq b$ and (when $\beta \neq 0$ ) such that $y^{\prime}(b) / y(b)>\beta^{\prime} / \beta$.

* This identity follows from the following fact :

If when $a \leqq x \leqq b$ and $L>\lambda>l, \phi(x, \lambda)$ is a continuous function of $(x, \lambda)$; and if when $L>\lambda^{\prime}>\lambda^{\prime \prime}>l, \phi\left(x, \lambda^{\prime}\right) \geqq \phi\left(x, \lambda^{\prime \prime}\right)$; and if for every value of $x$ in the interval $a \leqq x \leqq b \lim _{\lambda=L} \phi(x, \lambda) \equiv+\infty ;$ then no matter how

large a positive quantity $M$ we may choose it is possible to find a quantity $K$ such that when $L>\lambda>K$ and $a \leqq x \leqq b, \phi(x, \lambda)>M$.

The idea contained in this theorem might be expressed by saying that $\phi(x, \lambda)$ diverges towards $+\infty$ uniformly throughout the interval $a \leqq x \leqq b$. As I am unable to refer to any place where this theorem is proved I will give a proof of it here.

Take an infinite number of values $\lambda^{\prime}, \lambda^{\prime \prime}, \lambda^{\prime \prime \prime}, \ldots$ such that $l<\lambda^{\prime}<\lambda^{\prime \prime}$ $<\ldots<L$ and such that $\lim \lambda^{[n]}=L$. "Then we have $\phi\left(x, \lambda^{\prime}\right)<\phi\left(x, \lambda^{\prime \prime}\right)$ $<\ldots$ and $\lim _{n=\infty} \phi\left(x, \lambda^{[n]}\right) \stackrel{n=\infty}{=}+\infty$. Now let $x_{n}$ be that value (or one of the values) of $x$ in the interval $a \leqq x \leqq b$ at which $\phi\left(x, \lambda^{[n]}\right)$ is smallest. Then either $\lim _{n=\infty} \phi\left(x_{n}, \lambda[n]\right)=+\infty$, in which case our theorem is evidently true, or we can find a positive quantity $M$ so large that no matter how large $n$ is $\phi\left(x_{n}, \lambda[n]\right)<M$. To show that this last case is impossible let us consider the points $x_{1}, x_{2}, x_{3}, \ldots$. These points must have at least one limiting point in the interval $a \leqq x \leqq b$. Let $\bar{x}$ be such a limiting point. Now by hypothesis it is possible to take $\lambda$ so near to $L$ that $\phi(\bar{x}, \lambda)>M$. Suppose then that when $n>n_{1}, \phi\left(\bar{x}, \lambda^{[n]}\right)>M$. Since $\phi\left(x, \lambda^{[n]}\right)$ is continuous at $\bar{x}$ it is possible to take the positive quantity $\varepsilon$ so small that throughout the interval from $\bar{x}-\varepsilon$ to $\bar{x}+\varepsilon \phi\left(x, \lambda^{\left[n_{1}\right]}\right)>M$, and accordingly when $n>n_{1}, \phi(x, \lambda[n])>M$ throughout this interval. But this is impossible since there are within this interval an infinite number of the points $x_{1}, x_{2}, \ldots$ at which $\phi\left(x_{n}, \lambda^{[n]}\right)<M$.

I will add that from this theorem may be deduced (or conversely that this theorem may be deduced from) the theorem that if a series whose terms are continuous functions converges throughout the interval $a \leqq x$ $\leqq b$ to a continuous limit and if the terms of the series in this interval are nowhere negative, the series is uniformly convergent. 
That we can find a quantity $l_{1}$ as described in $(\alpha)$ is obvious at once. For let $\alpha_{1}$ and $b_{1}$ be two points, such that $a \leqq a_{1}<b_{1} \leqq b$ but such that none of the points at which the formula $\lim _{\lambda=l} \varphi(x, \lambda)=-\infty$ fails to hold lie in the interval $a_{1} \leqq x \leqq b_{1}$. Then we can take $l_{1}$ so that when $l<\lambda \leqq l_{1}$ $\varphi(x, \lambda)<-\frac{(m+1)^{2} \pi^{2}}{(b-a)^{2}}\left(a_{1} \leqq x \leqq b_{1}\right)$ so that (Th. of Osc. 1 , Theorem V) every solution of (2) has at least $m+1$ roots in the interval $a_{1}<x<b_{1}$ and therefore $a$ fortiori in the interval $a<x<b$.

The proof that we can find a quantity $L_{1}$ as described in $(\beta)$ is less simple. Let us first consider the case in which the formula $\lim \varphi(x, \lambda)=+\infty$ holds at all points of the interval $a \leqq x \leqq b$ except at one or both of the extremities $a, b . *$ Let us denote by $y_{1}(x, \lambda)$ and $y_{2}(x, \lambda)$ the solutions of $(2)$ which satisfy respectively the relations :

$$
y_{1}(a, \lambda)=\alpha, y_{1}^{\prime}(\alpha, \lambda)=\alpha^{\prime} \text { and } y_{2}(b, \lambda)=\beta, y_{2}^{\prime}(b, \lambda)=\beta^{\prime} \text {. }
$$

Choose arbitrarily a fixed quantity $\bar{\lambda}$ such that $l<\bar{\lambda}<L$. Then since $y_{1}(x, \bar{\lambda})$ and $y_{2}(x, \bar{\lambda})$ each has at most a finite number of roots in the interval $a b$ it is possible to find a positive quantity $\varepsilon$ so small that letting $a+\varepsilon=a_{1}$ and $b-\varepsilon=b_{1} y_{1}(x, \bar{\lambda})$ has no root in the interval $a<x \leqq a_{1}$ and $y_{2}(x, \bar{\lambda})$ has no root in the interval $b_{1} \leqq x<b$. It follows by the first theorem of comparison (Th. of Osc. 1, Theorem I) that when $\bar{\lambda} \leqq \lambda<L, y_{1}(x, \lambda)$ and $y_{2}(x, \lambda)$ have no roots in the intervals $a<x \leqq a_{1}$ and $b_{1} \leqq x<b$ respectively. By the second theorem of comparison (Th. of Osc. 1, Theorem II) it follows that when $\bar{\lambda}<\lambda<L$ :

$$
\frac{y_{1}^{\prime}\left(a_{1}, \lambda\right)}{y_{1}\left(a_{1}, \lambda\right)}>\frac{y_{1}^{\prime}\left(a_{1}, \bar{\lambda}\right)}{y_{1}\left(a_{1}, \lambda\right)} \text { and } \frac{y_{2}^{\prime}\left(b_{1}, \lambda\right)}{y_{2}\left(b_{1}, \lambda\right)}<\frac{y_{2}^{\prime}\left(b_{1}, \bar{\lambda}\right) \dagger}{y_{2}\left(b_{1}, \bar{\lambda}\right)} \text {. }
$$

If now we denote by $\bar{y}(x, \lambda)$ the solution of (2) for which $\bar{y}\left(a_{1}, \lambda\right)=y_{1}\left(a_{1}, \bar{\lambda}\right)$ and $\bar{y}^{\prime}\left(a_{1}, \lambda\right)=y_{1}^{\prime}\left(a_{1}, \bar{\lambda}\right)$ (so that when $\lambda=\bar{\lambda}, y_{1}$ and $\bar{y}$ are identical), we see (Th. of Osc. 1, Theorems III and IV) that it is possible to find a quantity

* This is, in fact, the only case we shall make any application of in this paper. The more general case would, however, be necessary in the problem mentioned in the third footnote on p. 376 .

$\dagger$ The reversal of the sign of inequality here is due to the fact that in order to apply the theorem of comparison we must introduce the new variable $x^{\prime}=-x$ and this reverses the sign of $y_{2}{ }^{\prime}$. 
$L_{1}\left(\bar{\lambda}<L_{1}<L\right)$ such that when $L_{1} \leqq \lambda<L, \bar{y}(x, \lambda)$ has no roots in the interval $a_{1} \leqq x \leqq b_{1}$ and $\bar{y}^{\prime}\left(b_{1}, \lambda\right) / \bar{y}\left(b_{1}, \lambda\right)$ $>y_{2}{ }^{\prime}\left(b_{1}, \bar{\lambda}\right) / y_{2}\left(b_{1}, \bar{\lambda}\right)$. Let us now compare $y_{1}$ and $\bar{y}$ with each other by applying Theorems I and II of the present paper to the interval $a_{1} \leqq x \leqq b_{1}$. We see that when $L_{1}<\lambda<L, y_{1}(x, \lambda)$ has no roots in the interval $a_{1} \leqq x \leqq b_{1}$ (and therefore none in the interval $a<x \leqq b_{1}$ ) and that $y_{1}^{\prime}\left(b_{1}, \lambda\right) / y_{1}\left(b_{1}, \lambda\right)>y_{2}^{\prime}\left(b_{1}, \bar{\lambda}\right) / y_{2}\left(b_{1}, \bar{\lambda}\right)>y_{2}^{\prime}\left(b_{1}, \lambda\right) / y_{2}\left(b_{1}, \lambda\right)$. Finally applying the same theorems again to the interval $b_{1} \leqq x \leqq b$ so as to compare $y_{1}$ and $y_{2}$ we see that when $L_{1} \leqq \lambda<L, y_{1}(x, \lambda)$ has no roots in this interval (and therefore none in the interval $a<x<b$ as was to be proved), and that: $y_{1}^{\prime}(b, \lambda) / y_{1}(b, \lambda)>y_{2}^{\prime}(b, \lambda) / y_{2}(b, \lambda)=\beta^{\prime} / \beta$ (as was also to be proved).

If now the formula $\lim \varphi(x, \lambda)=+\infty$ fails to hold at the

points $c_{1}, c_{2}, \cdots, c_{k}$, as well as perhaps at the extremities $a, b$ of the interval, we can easily prove the existence of the quantity $L_{1}$ described in $(\beta)$ by breaking up the interval $a b$ into sub-intervals $a c_{1}, c_{1} c_{2}, \cdots, c_{k} b$. Then it is clear from the special case we have just treated that we can find constants $L^{\prime}, L^{\prime \prime}, L^{\prime \prime \prime}, \cdots, L^{[k+1]}$ such that :

(1) when $L^{\prime} \leqq \lambda<L$ the solution of (2) for which $y(\alpha, \lambda)=\alpha$ and $y^{\prime}(\alpha, \lambda)=\alpha^{\prime}$ has no root in the interval $a<x \leqq c_{1}$ and satisfies the relation $y^{\prime}\left(c_{1}, \lambda\right) / y\left(c_{1}, \lambda\right)>1$;

(2) when $L^{[i]} \leqq \lambda<L(i=2,3, \cdots, k)$ the solution of (2) for which $y^{\prime}\left(c_{i-1}, \lambda\right) / y\left(c_{i-1}, \lambda\right)=1$ has no root in the interval $c_{i-1} \leqq x \leqq c_{i}$ and satisfies the relation $y^{\prime}\left(c_{i}, \lambda\right) / y\left(c_{i}, \lambda\right)>1$;

(3) when $L^{[k+1]} \leqq \lambda<L$ the solution of (2) for which $y^{\prime}\left(c_{k}, \lambda\right) / y\left(c_{k}, \lambda\right)=1$ has no root in the interval $c_{k} \leqq x \leqq b$ and (when $\beta \neq 0$ ) satisfies the relation

$$
y^{\prime}(b, \lambda) / y(b, \lambda)>\beta^{\prime} / \beta .
$$

If we let $L_{1}$ be the largest of the quantities $L^{\prime}, L^{\prime \prime}$, $\cdots, L^{[k+1]}$ the truth of $(\beta)$ follows at once from Theorems $I^{\text {. }}$ and II of the present paper.

\section{\$2. Klein's Generalized Theorem of Oscillation.}

We start here from the differential equation (1) in which we will suppose the coefficient $q$ to have the form :

$$
q(x)=\chi(x)+\psi^{\prime}(x)\left[C_{k} x^{k}+C_{k-1} x^{k-1}+\cdots+C_{1} x+C_{0}\right],
$$

and we will suppose that the functions $p(x), \chi(x)$, and $\psi(x)$ 
do not involve the parameters $C_{0}, C_{1}, \cdots, C_{k}$. Let there be given $k+1$ finite intervals $a_{0} b_{0}, a_{1} b_{1}, \cdots, a_{k} b_{k}$ such that:

$$
a_{0}<b_{0} \leqq a_{1}<b_{1} \leqq a_{2}<b_{2} \cdots<b_{k-1} \leqq a_{k}<b_{k},
$$

and let us assume that throughout each of the intervals $a_{i} \leqq x \leqq b_{i}(i=0,1, \cdots, k)$ the functions $p(x), \chi(x)$, and $\psi(x)$ are single valued, continuous, and real, and that $\psi(x)$ does not change sign in any one of these intervals nor have an infinite number of roots in them. Then we may state Klein's generalized theorem of oscillation as follows :

IV. There is one and only one real determination of the parameters $C_{0}, C_{1}, \cdots, C_{k}$ for which the equation (1) has $k+1$ solutions $y_{0}, y_{1}, \cdots, y_{k}$ such that $y_{i}(i=0,1, \cdots, k)$ has just $m_{i}$ roots in the interval $a_{i}<x<b_{i}$ and satisfies the conditions :

$$
\begin{aligned}
& \alpha_{i}^{\prime} y_{i}\left(a_{i}\right)-\alpha_{i} y_{i}^{\prime}\left(a_{i}\right)=0, \\
& \beta_{i}^{\prime} y_{i}\left(b_{i}\right)-\beta_{i} y_{i}^{\prime}\left(b_{i}\right)=0 .
\end{aligned}
$$

In this theorem the quantities $m_{i}$ are any integers positive or zero and $\alpha_{i}, \alpha_{i}^{\prime}, \beta_{i}, \beta_{i}^{\prime}$ are any real quantities subject only to the restriction that $\alpha_{i}$ and $\alpha_{i}^{\prime}$ are not both zero and that $\beta_{i}$ and $\beta_{i}^{\prime}$ are not both zero.

In order to prove the above theorem we will introduce the variables:

$$
t_{i}=g_{i} \int_{\xi_{i}}^{x} e^{-\int_{\xi_{i}}^{x} d x} d x+f_{i} \quad(i=0,1, \cdots, k)
$$

where $g_{i}$ and $f_{i}$ are real constants which may be arbitrarily chosen provided $g_{i} \neq 0$, and $\xi_{i}$ is any real constant subject to the condition $a_{i} \leqq \xi_{i} \leqq b_{i}$. It should be noticed that the variable $t_{i}$ as thus defined is real when $\alpha_{i} \leqq x \leqq b_{i}$, and that this interval of the $x$-axis corresponds in a one to one manner to a finite interval $\sigma_{i} \tau_{i}$ of the $t_{i}$-axis. Written in terms of $t_{i}$, (1) has the form :

$$
\frac{d^{2} y}{d t_{i}^{2}}=\varphi_{i}(x) y
$$

where :

$$
\varphi_{i}(x)=\frac{-1}{g_{i}^{2}} \chi(x) e^{2 \sum_{\xi_{i}}^{x} d x}-\frac{1}{g_{i}^{2}} \psi(x) e^{2 \int_{\xi_{i}}^{x} d x}\left[C_{h} x^{k}+\cdots+C_{0}\right] .
$$

It is important to bear in mind in the following work that if a solution $y_{i}$ of (1) regarded as a function of $x$ has $m_{i}$ roots in the interval $a_{i}<x<b_{i}$ and satisfies the two con- 
ditions (3), the same function regarded as a function of $t_{i}$ will be a solution of (4) having $m_{i}$ roots in the interval $\sigma_{i}<t_{i}<\tau_{i}$ (or $\tau_{i}<t_{i}<\sigma_{i}$ ) and will satisfy relations of the form :

$$
\begin{aligned}
& \alpha_{i}^{\prime} y_{i}\left(\sigma_{i}\right)-\bar{\alpha}_{i} y_{i}^{\prime}\left(\sigma_{i}\right)=0, \\
& \beta_{i}^{\prime} y_{i}\left(\tau_{i}\right)-\bar{\beta}_{i} y_{i}^{\prime}\left(\tau_{i}\right)=0,
\end{aligned}
$$

where $y^{\prime}=\frac{d y}{d t_{i}}$ and where $\overline{\alpha_{i}}$ and $\bar{\beta}_{i}$ are constant (not zero) multiples of $\alpha_{i}$ and $\beta_{i}$; and that the converse of the statement just made is also true.

It is easily seen that if $k=0$ Theorem IV is merely a special case of Theorem III since then if $\psi$ is negative in the interval $a_{0} b_{0} \varphi_{0}(x)$ will continually increase (or rather never decrease) for every point of this interval as $C_{0}$ increases from $-\infty$ to $+\infty$ and will, except for the points where $\psi(x)=0$, increase from $-\infty$ to $+\infty$; while if $\psi$ is positive the same will be true as $-C_{0}$ increases from $-\infty$ to $+\infty$. Theorem IV is therefore true if there is only one parameter and one interval.

Let us then, following the ordinary method of mathematical induction, assume that Theorem IV is true for $k$ parameters and $k$ intervals. If from this assumption we can deduce the theorem for $k+1$ parameters and intervals the proof of the theorem will be complete.

Since in (1) we may write :

where

$$
\begin{gathered}
q(x)=\bar{\chi}(x)+\psi(x)\left[C_{k-1} x^{k-1}+\cdots+C_{0}\right], \\
\bar{\chi}(x)=\chi(x)+C_{k} x^{k} \psi(x),
\end{gathered}
$$

it follows (from the assumption we have made that our theorem holds when there are $k$ parameters) that, $C_{k}$ having an arbitrarily assigned fixed value, there is always one and only one real set of values $C_{0}, C_{1}, \cdots, C_{k-1}$ for which (1) has $k$ solutions $y_{0}, y_{1}, \cdots, y_{k-1}$ satisfying in the intervals $a_{i} b_{i}(i=0,1$, $\cdots, k-1)$ the desired conditions. From this point on we will assume that $C_{0}, C_{1}, \cdots, C_{k-1}$, have thus been determined as single valued real functions of $C_{k}$. It remains then merely to show that there is one and only one value of $C_{k}$ for which (1) has a solution $y_{k}$ which satisfies the desired conditions in the interval $a_{k} b_{k}$.

In order to indicate the dependence of $\varphi_{i}$ on $C_{k}$ we will write $\varphi_{i}\left(x, C_{k}\right)$. Let us now assign to $C_{k}$ two different values $C_{k}^{\prime}$ and $C_{k}^{\prime \prime}$ and consider the difference : 


$$
\begin{gathered}
\varphi_{i}\left(x, C_{k}^{\prime}\right)-\varphi_{i}\left(x, C_{k}^{\prime \prime}\right) \\
=\frac{-1}{g_{i}^{2}} \psi(x) e^{2 \sum_{\xi_{i}}^{x} x}\left[\left(C_{k}^{\prime}-C_{k}^{\prime \prime}\right) x^{k}+\cdots+\left(C_{0}^{\prime}-C_{0}^{\prime \prime}\right)\right] .
\end{gathered}
$$

Since by hypothesis each of the equations :

$$
\frac{d^{2} y}{d t_{i}^{2}}=\varphi_{i}\left(x, C_{k}^{\prime}\right) y \text { and } \frac{d^{2} y}{d t_{i}^{2}}=\varphi_{i}\left(x, C_{k}^{\prime \prime}\right) y
$$

has a solution with $m_{i}$ roots in the interval $\sigma_{i}<t_{i}<\tau_{i}$ $(i=0,1, \cdots, k-1)$ and since these two solutions satisfy the same conditions (5) it follows at once from the theorems of comparison ( $T h$. of Osc. 1, Theorems I and II) that the above difference must change sign in the interval $\sigma_{i}<t_{i}<\tau_{i}$ that is in the interval $a_{i}<x<b_{i}$. It follows that the polynomial :

$$
\left(C_{k}^{\prime}-C_{k}^{\prime \prime}\right) x^{k}+\left(C_{k-1}^{\prime}-C_{k-1}^{\prime \prime}\right) x^{k-1}+\cdots+\left(C_{0}^{\prime}-C_{0}^{\prime \prime}\right)
$$

must have a root $x_{i}$ in this interval. Accordingly when $i=0,1, \cdots, k$ :

$$
\varphi_{i}\left(x, C_{k}^{\prime}\right)-\varphi_{i}\left(x, C_{k}^{\prime \prime}\right)
$$

$=\frac{-1}{g_{i}{ }^{2}} \psi(x) e^{2 \int_{\xi_{i}}^{x} d x}\left(C_{k}^{\prime}-C_{k}^{\prime \prime}\right)\left(x-x_{0}\right)\left(x-x_{1}\right) \cdots\left(x-x_{k-1}\right)$,

where $a_{j}<x_{j}<b_{j}(j=0,1, \cdots, k-1)$. From this fundamental formula we can deduce several important results :

(a) $\varphi_{k}\left(x, C_{k}\right)$ is when $\alpha_{k} \leqq x \leqq b_{k}$ and for all values of $C_{k}$ a continuous function of $\left(x, C_{k}\right)$ and therefore of $\left(t_{k}, C_{k}\right)$.

For in the first place it is clear that it is a continuous function of $x$, so that however small the positive quantity $\varepsilon$ may be, we can choose the positive quantity $\delta_{1}$ so small that when $\left.|x-\bar{x}| \leq \delta_{1}, \mid \varphi_{k} \overline{(x}, C_{k}\right)-\varphi_{k}\left(x, C_{k}\right) \mid \leq \varepsilon / 2$. Moreover from (6) $\left|\varphi_{k}\left(\bar{x}, C_{k}\right)-\varphi_{k}\left(\bar{x}, \overline{C_{k}}\right)\right|<K\left|C_{k}-\bar{C}_{k}\right|$ where $K$ is a positive constant, independent of $\bar{x}, C_{k}$ and $\bar{C}_{k} *$ From the last formula it is clear that however small $\varepsilon$ may be, we can choose a positive quantity $\delta_{2}$ so small that when

$$
\left.\left|C_{k}-\bar{C}_{k}\right|<\delta_{2}, \quad \mid \varphi_{k} \overline{(x}, C_{k}\right)-\varphi_{k}\left(\bar{x}, \overline{C_{k}}\right) \mid<\varepsilon / 2 .
$$

* We need merely to take $K$ greater than the greatest value of :

$$
\frac{1}{g_{k}^{2}}|\psi(x)| e^{2 \int_{\xi_{k}}^{x} d x}\left(x-a_{0}\right)\left(x-a_{1}\right) \ldots\left(x-a_{k-1}\right)
$$

in the interval $a_{k} \leqq x \leqq b_{k}$. 
Accordingly letting $\delta$ denote the smaller of the two quantities $\delta_{1}$ and $\delta_{2}$ we see that when

$|x-\bar{x}|<\delta$ and $\left.\left|C_{k}-\bar{C}_{k}\right|<\delta, \quad \mid \varphi_{k}\left(x, C_{k}\right)-\varphi_{k} \overline{(x}, \overline{C_{k}}\right) \mid<\varepsilon$ that is $\varphi_{k}\left(x, C_{k}\right)$ is a continuous function of $\left(x, C_{k}\right)$ as was to be proved.

( $\beta$ ) If $C_{k}^{\prime}>C_{k}^{\prime \prime}$ then $\varphi_{k}\left(x, C_{k}^{\prime}\right) \geqq \varphi_{k}\left(x, C_{k}^{\prime \prime}\right) *\left(a \leqq x \leqq b_{k}\right)$ provided that $\psi(x)$ is negative in this interval; otherwise $\varphi_{k}\left(x, C_{k}^{\prime}\right) \leqq \varphi_{k}\left(x, C_{k}^{\prime \prime}\right)$.*

(r) Except at the points at which $\phi(x)=0$ and perhaps the point $x=a_{k}$ we have at every point $x$ of the interval $a_{k} \leqq x \leqq b_{k}$ : $\lim _{C_{k}} \varphi_{k}\left(x, C_{k}\right)=\infty$. $C_{k}=\infty$

The truth of $(\beta)$ and $(\gamma)$ follows immediately from (6).

Accordingly $\varphi_{k}\left(x, C_{k}\right)$ is at every point of the interval $a_{k} \leqq x \leqq b_{k}$, i. e., of $\sigma_{k} \leqq t_{k} \leqq \tau_{k}$, a continuous function of $\left(t_{k}, C_{k}\right)$ which, except at a finite number of points, continually increases with $C_{k}$ (or $-C_{k}$ ), and except at a finite number of points increases from $-\infty$ to $+\infty$ as $C_{k}\left(\right.$ or $\left.-C_{k}\right)$ varies from $-\infty$ to $+\infty$. Sturm's generalized theorem of oscillation (Theorem III) tells us therefore that there is one and only one value of $C_{k}$ for which (4) has a solution satisfying the desired conditions in the interval $\sigma_{k} \tau_{k}$ and the proof of Theorem IV is complete.

We proceed now to a generalization of the preceding theorem :

V. In Theorem IV we may at some or at all of the points $a_{i}$, $b_{i}$ drop the requirement that the functions $p(x), \chi(x)$, and $\psi(x)$ $b$ continuous provided that as $x$ approaches these points the functions $t_{i}$ and $\varphi_{i}$ approach finite limits. $\dagger$ We must, however, then at these points replace conditions (3) by conditions (5), the two sets of conditions being then no longer equivalent.

It is not necessary to give any new proof of this theorem as the proof just given of Theorem IV applies at once since what is essential in that proof referred not to the form (1) of the differential equation but to the binomial form (4).

We proceed now to recall certain important special cases of (1) to which Theorems IV and V apply:

* The equality sign holding at most for a finite number of values of $x$ namely for the points if any at which $\psi(x)=0$.

$\dagger \mathrm{I}$ should like to add here that we need not even require that the limit of $\phi_{i}$ be finite, all that is necessary being that $\phi_{i}$ do not become infinite too rapidly as $x$ approaches $a_{i}$ and $b_{i}$. The exact statement of the conditions here as well as all proof I must, however, reserve for a future occasion. 
The most general homogeneous linear differential equation of the second order with rational coefficients which is everywhere regular and has as its finite singular points the points $e_{i}(i=1,2, \cdots, n)$ with exponents $x_{i}^{\prime}, x_{i}^{\prime \prime}$ is :*

$$
\begin{aligned}
\frac{d^{2} y}{d x^{2}} & +\left(\frac{1-x_{1}^{\prime}-x_{1}^{\prime \prime}}{x-e_{1}}+\cdots+\frac{1-x_{n}^{\prime}-x_{n}^{\prime \prime}}{x-e_{n}}\right) \frac{d y}{d x} \\
& +\frac{1}{f(x)}\left[\frac{x_{1}^{\prime} x_{1}^{\prime \prime} f^{\prime}\left(e_{1}\right)}{x-e_{1}}+\cdots+\frac{x_{n}^{\prime} x_{n}^{\prime \prime} f^{\prime}\left(e_{n}\right)}{x-e_{n}}\right. \\
& \left.+C_{n-2} x^{n-2}+C_{n-3} x^{n-3}+\cdots+C_{0}\right] y=0
\end{aligned}
$$

where

$$
f(x)=\left(x-e_{1}\right)\left(x-e_{2}\right) \cdots\left(x-e_{n}\right)
$$

and $f^{\prime}(x)=d f(x) / d x$. The point $x=\infty$, the product of whose exponents is $C_{n-2}$, may be singular or non-singular.

We will suppose that the coefficients of this equation are real for real values of $x$. For this to be the case it is necessary and sufficient that:

(a) the singular points $e_{1}, e_{2}, \cdots, e_{n}$ be conjugate imaginaries in pairs in so far as they are not real ;

(b) the exponents $x_{i}^{\prime}, x_{i}^{\prime \prime}$ of a real singular point $e_{i}$ be either real or conjugate imaginaries ;

(c) the exponents $x_{i}^{\prime}, x_{i}^{\prime \prime}$ and $x_{j}^{\prime}, x_{j}^{\prime \prime}$ of two conjugate imaginary singular points $e_{i}$ and $e_{j}$ be so related that $x_{i}^{\prime}$ is the conjugate of one and that $x_{i}^{\prime \prime}$ is the conjugate of the other of the two quantities $x_{j}^{\prime}, x_{j}^{\prime \prime}$;

(d) the quantities $C_{n-2}, C_{n-3}, \cdots, C_{0}$ be real.

Let us now take $k+1$ intervals $a_{i} b_{i}(i=0,1, \cdots, k)$ on the real $x$-axis as on p. $371, i$. e., so that no two of these intervals overlap and none of the points $e_{1}, e_{2}, \cdots, e_{n}$ lie within or at an extremity of any of these intervals: If we suppose that $k \leqq n-2$ we see that we can determine the parameters $C_{k}, C_{k-1}, \cdots, C_{0}^{\gamma}$ by means of the Theorem of Oscillation IV.

Let us now assume that in equation (7) one of the singular points, which for the sake of simplicity we will take as $e_{1}$, is real and that $x_{1}^{\prime \prime}=0$ while $x_{1}^{\prime}$ is real and satisfies the inequality $0 \leqslant x_{1}^{\prime} \supseteqq \frac{1}{2}$. Then if one of the intervals $a_{i} b_{i}$ has an extremity at $e_{1}$, we see that $t_{i}$ and $\varphi_{i}$ approach finite limits as $x$ approaches $e_{1}$, so that we see by Theorem $\mathrm{V}$ that we can still determine the parameters $C_{k}, \mathrm{C}_{k-1}^{\prime}, \cdots, C_{0}$ by means of the theorem of oscillation.

* Cf. for instance my book: "Ueber die Reihenentwickelungen der Potentialtheorie," pp. 112-113. 
It goes without saying that any number of the extremities of the intervals $\alpha_{i} b_{i}(i=0,1, \cdots, k)$ may lie at singular points provided that the conditions above stated for the point $e_{1}$ are satisfied at each of these points.

It should be noticed that the generalized Lamé's equation, whether looked at from Heine's* or from Klein's $\dagger$ standpoint, has as the exponents of each of its finite singular points the values $0, \frac{1}{2}$, so that in this case any or all of the intervals $a_{i} b_{i}$ may reach up to singular points.

We will note in conclusion that the cases we have just mentioned by no means exhaust the important applications of Theorems IV and V. For instance, the degenerate forms of Lamé's equation where two or more singular points coincide come immediately under Theorem IV.

HaRVARD University, CAMBridge, Mass.

\section{THE CONSTRUCTION OF SPECIAL REGULAR RETICULATIONS ON A CLOSED SURFACE.}

BY PROFLSSOR HENRY S. WHITE.

(Read in part at the Mathematical Conference in Chicago, January 1, 1897, and in final form at the Meeting of the American Mathematical Society, April 30, 1898.)

\section{INTRODUCTORY.}

The reticulations whose existence is here to be discussed are called regular because of two properties : the number of termini of edges assembled in one vertex is the same for all vertices of the reticulation, and the number of edges in the boundary of a face is the same for all faces. These two numbers, $r$ and $s$, together with $p$, the deficiency of the supporting surface, shall be assumed to characterize the reticulation sufficiently for present purposes. Of regular reticulations classified on this basis, only a finite number of classes are possible on a surface of given deficiency. Some of these possible classes, if $p>2$, are derivable from those of lower deficiency; those not so derivable are properly

* Handbuch der Kugelfunctionen, vol. I., p. 445.

†Cf. my book: "Ueber die Keihenentwickelungen der Potentialtheorie," p. 114.

$\ddagger$ It is in fact easy to see that they may turn back at the singular points and thus cover parts of the $x$-axis more than once. Cf. p. 123 of my book just referred to. 